\title{
Lumen
}

Selected Proceedings from the Canadian Society for Eighteenth-Century Studies

\section{Deux facettes de l'ennui chez Madame de Graffigny : de l'ennui quotidien à l'ennui tragique}

\section{Raoudha Kallel}

Volume 27, 2008

North America at the Crossroads of European Cultures in the Eighteenth Century

L’Amérique du Nord au Carrefour des cultures au XVIII ${ }^{\mathrm{e}}$ siècle

URI : https://id.erudit.org/iderudit/1012055ar

DOI : https://doi.org/10.7202/1012055ar

Aller au sommaire du numéro

Éditeur(s)

Canadian Society for Eighteenth-Century Studies / Société canadienne d'étude du dix-huitième siècle

ISSN

1209-3696 (imprimé)

1927-8284 (numérique)

Découvrir la revue

Citer cet article

Kallel, R. (2008). Deux facettes de l'ennui chez Madame de Graffigny : de l'ennui quotidien à l'ennui tragique. Lumen, 27, 137-155.

https://doi.org/10.7202/1012055ar

Copyright (c) Canadian Society for Eighteenth-Century Studies / Sociéte canadienne d'étude du dix-huitième siècle, 2009
Ce document est protégé par la loi sur le droit d'auteur. L'utilisation des services d'Érudit (y compris la reproduction) est assujettie à sa politique d'utilisation que vous pouvez consulter en ligne.

https://apropos.erudit.org/fr/usagers/politique-dutilisation/ 


\section{Deux facettes de l'ennui chez Madame de Graffigny: de l'ennui quotidien à l'ennui tragique}

"Ah, mon Dieu, je suis outrée, excédée, morte, trépassée d'ennui. Cela ne fait que croître et embellir ${ }^{1} »$. C'est ainsi que Mme de Graffigny apparaît lorsqu'elle est torturée par l'ennui. Comme plusieurs de ses contemporaines, elle est touchée par ce mal, que l'écriture de romans et la compagnie des gens d'esprit ne l'ont pas vraiment aidée à combattre. Ce mal, elle ne cesse de le maudire ou de le dénoncer. Dans sa Correspondance, c'est le leitmotiv qui embellit ses plaintes et ses mécontentements; dans sa vie, c'est la douleur par excellence qui décore son quotidien et flétrit son univers. Mme de Graffigny est, à coup sûr, une créature d'ennui. Si l'ennui, chez elle, est un mal qui se définit comme "l'opposé et le destructeur du plaisir ${ }^{2}$ ", il est aussi une obsession. À cet égard, l'ennui domine, d'une façon évidente, son esprit et son écriture; il enrichit son lexique épistolaire:

Reprenons le fil de ma vie, peut-être aussi ennuyeuse à lire qu'à filer. Je fus hier un moment chez la belle. Elle allait sortir. Je remontai dans l'ennuyeuse chambre de mon ennuyeuse malade. J'y fis mon ennuyeuse contenance à souper, car je

1 Correspondance de Madame de Graffigny, édition préparée par English Showalter avec la collaboration de P. Allan, P. Bouillaguet, N. Boursier, J. Curtis, J. A. Dainard, L. C. Kerslake, D. W. Smith, D. A. Trott et E. A. Walker, Oxford, Voltaire Foundation, 1985, tome I, Lettre 58, p. 184. Désormais, les références à la correspondance, dans le texte ou en note, se feront comme suit: numéro du volume (en chiffres romains), numéro de la lettre, numéro de la page. Pour une étude thématique et chronologique de corpus, voir J. A. Dainard et al., «La Correspondance de Madame de Graffigny", Dix-Huitième siècle, nº10, 1978, 379-394.

2 II, L. 198, p. 199. 
ne mange pas. Il m'est impossible d'écrire les soirs. Je suis plus fatiguée d'ennui que je ne le serais d'une course à cheval. Je me suis levée à sept heures. [...] Mon Dieu, mes chers amis, je n'avais pas besoin d'être excédée d'ennui pour vous regretter, mais je ne souffre jamais un mal tout seul. ${ }^{3}$

La vie est ennuyeuse. La chambre est ennuyeuse. La malade est ennuyeuse. Sans doute, tout est-il stigmatisé par l'ennui aux yeux de Mme de Graffigny. Ce "profond ennui » est en effet ancré dans chaque mouvement, chaque activité, chaque geste, chaque conversation. ${ }^{4}$ Mme de Graffigny est hantée par l'ennui ; «[i]nvisible maladie qui tient simultanément de l'être et du non-être», ${ }^{5}$ l'ennui l'accompagne partout, implicitement ou explicitement. Elle se lève avec l'ennui ; elle se couche avec lui. Aucun dîner, aucun souper, aucune visite n'est en mesure d'effacer les traces de cette maladie obsédante. Mme de Graffigny est victime d'un "ennui mortel» ${ }^{6}$ qui lui gâche la matinée et la soirée: ${ }^{7}$ " aujourd'hui la matinée s'est passée comme à l'ordinaire, c'est-à-dire avec l'ennui du soir $»^{8}$ " $[$ A] $]$ ssez uniformes» ${ }^{9}$ et vides d'occupations, ses

3 I, L. 31, p. 49. Ce paragraphe témoigne aussi de la richesse du lexique de l'ennui chez Mme de Graffigny: "Ah, que je me suis ennuyée hier, mon pauvre ami. Javote avait des vapeurs, et des vapeurs les plus ennuyeuses que jamais on ait eu[es]. Nous allâmes faire une visite chez une dame où j'avais fait un souper si ennuyeux : la visite fut ennuyeuse. [...] Dès que nous fûmes revenues, le D. vint. Il harpouilla l'ennuyeuse [...] » (II, L. 161, p. 77).

4 III, L. 354, p. 92.

5 Vladimir Jankélévitch, L'Alternative, Paris, Librairie Félix Alcan, 1938, p. 126.

6 Georges Bérubé, «Mme de Graffigny à Cirey : écrire pour exister 'par procuration' ", dans Marie-France Silver et Marie-Laure Girou Swiderski (éd.), Femmes en toutes lettres : les épistolières du XVIII siècle, Oxford, Voltaire Foundation, 2000, p. 24.

7 À cet égard, l'analyse de l'ennui chez Émile Tardieu est intéressante. L'auteur y distingue trois formes d'ennui au cours de la journée : l'ennui du matin, l'ennui de l'après-midi et l'ennui du soir : « L'ennui du matin est un voile sur nos facultés qui s'éveillent lentement [...]. L'ennui de l'après-midi vient de la durée de cette portion du jour, incommensurable à notre impatience [...]. L'ennui du soir a sa source dans la fatigue du jour qui nous livre désarmés à l'assaut des pensées tristes, tandis qu'elle gâte les plaisirs douteux que nous allons chercher dans les ténèbres " (L'ennui : étude psychologique, Paris, Alcan, 1903, p. 238-240).

8 III, L. 470, p. 398.

9 «Comme mes journées sont assez uniformes, je te vais les dire » (II, L. 193, p. 181). Il faut dire que Mme de Graffigny se plaint sans cesse de l'uniformité de ses journées : "Je ne te rends point compte de mes jours. Quand il n'y aurait que leur uniformité, cela serait ennuyeux » (I, L. 44, p. 113). 
journées sont uniquement remplies d'ennui : «Ma journée a été destinée de toute éternité au plus grand profond ennui». ${ }^{10}$ Décrire ses journées revient donc à décrire un mal qui l'étouffe et la tourmente inlassablement. L'ennui acquiert une signification quotidienne, il fait partie du programme de la journée, au même titre que le jeu et les visites. En fait, bien souvent Mme de Graffigny détermine ce programme en fonction de l'intensité de son malaise: " Je fais après souper comme les visites, je reste ou je m'en vais selon l'ennui ". ${ }^{11}$ L'ennui cesse d'être un mal psychique pour devenir un "ennui ordinaire» ${ }^{12}$ et circonstanciel qui, comme l'explique Vladimir Jankélévitch,

se cherche volontiers de bonnes raisons dans les circonstances [...]; ces circonstances en effet le favorisent [...]. [Cet] ennui est au centre d'un univers mince et diaphane où toutes choses s'enveloppent du voile gris de l'indifférence. [...] [L'ennui] ne raisonne que sur les accidents qui lui donnent raison. Tout lui est prétexte. $^{13}$

Le cas de Mme de Graffigny offre l'exemple d'un mal qui tire sa force de la réalité vécue, immédiate. Son rythme correspond à celui de ses journées. Son intensité dépend du degré du vide journalier dont il se nourrit. À cet égard, loin d'être limité par le temps, l'ennui de Mme de Graffigny affecte le passé, le présent et le futur. Il empoisonne l'hier et l'avant-hier: "Ah, que je me suis ennuyée hier, mon ami $»,{ }^{14}$ " Tu sais comme je me suis ennuyée avant-hier $» .{ }^{15}$ Il contamine aussi l'aujourd'hui et le lendemain : "Je me suis ennuyée hier et je m'ennuie aujourd'hui », ${ }^{16}$ "Demain, j'ai un relais d'ennui tout pur ${ }^{17}{ }^{17}$ Ici s'exprime clairement la caractéristique temporelle de l'ennui graffignien. Loin d'être un ennui abstrait, il s'agit d'un ennui réel, qui existe

10 I, L. 144, p. 550. «La journée d'aujourd'hui n'a pas été si ennuyeuse que celle d'hier » (I, L. 39, p. 84) ; " Je m'étais ennuyée complètement toute la journée » (I, L. 123, p. 461).

11 II, L. 193, p. 181.

12 C'est le titre du livre de Véronique Nahoum-Grappe, L'Ennui ordinaire : essai de phénoménologie sociale, Paris, Austral, 1995.

13 Vladimir Jankélévitch, L'Alternative, op. cit., p. 127.

14 II, L. 255, p. 355.

15 II, L. 151, p. 27.

16 IV, L. 553, p. 251.

17 III, L. 480 , p. 435. 
et évolue dans l'ici et le maintenant. ${ }^{18} \mathrm{Si}$ le temps sert à le concrétiser, il permet aussi de le dramatiser. À vrai dire, le temps qui est régi par l'ennui paraît interminable : "Il me semble qu'il y a mille ans que je ne $t^{\prime}$ ai rien dit. C'est que le temps double quand on s'ennuie $»{ }^{19}$

Il existe donc une forte corrélation entre «temps » et «ennui ». ${ }^{20}$ Dans ce contexte, la définition de Lavelle ne serait pas inappropriée : " $\left[l^{\prime}\right]$ ennui est $[. .$.$] par excellence le mal du temps.» { }^{21}$ Chez Mme de Graffigny, il est certain que le temps est générateur d'ennui. Cet ennui s'accompagne souvent d'une sensation de pesanteur ou de longueur. Et l'écriture de l'ennui qui est propre à cette vaporeuse abonde en expressions et en adverbes qui expriment le temps : "J'ai été hier chez Md. Duvigeon, comme je l'avais dit; j'y ai été longtemps et si longtemps que j'étais morte d'ennui [...] ». ${ }^{22}$ Ailleurs, elle ajoute : «Enfin, je deviendrai ce que je pourrais, mais quand je songe qu'autrefois je pleurais d'ennui quand j'avais passé trois heures avec La Taupe et que $j^{\prime} y$ ai déjà passé deux mois que j'y passerais encore je ne sais quel temps $[\ldots] »{ }^{23}$ Anne Wallemacq analyse le rapport qui existe entre temps et ennui, dans des termes qui décrivent parfaitement la situation de Mme de Graffigny :

Les minutes s'écoulent lentement, les heures s'allongent, de plus en plus épaisses, de plus en plus sirupeuses. Le temps ne passe plus, l'ennui s'installe, tenace, engluant. Le temps devient matière. Expérience commune, souvent peu

18 Louis Lavelle pense autrement lorsqu'il déclare : «L'ennui n'a pas de maintenant. Il est submergé par le passé. [...] Il cède toujours à la langueur des souvenirs. Ainsi, pour l'ennui il n'y pas d'avenir " (Psychologie et spiritualité, Paris, Albin Michel, 1967, p. 220).

19 I, L. 31, p. 47.

20 Richard W. Bargdill met en évidence cette corrélation : «Straus [...] suggests that every moment of existence is a moment of personal becoming, and thus boredom appears to be a blockage of that becoming. [...] In boredom and depression there is the experience of time standing still, and in both the future is 'rendered inaccessible.' The depressed is stuck looking back to the past and it appears that the bored person is stuck in the present 'which is no longer a continuation of the past'. " ("Being Bored with one's Life: An Empirical Phenomenological Study », thèse Duquesne University, 1998, p. 46.)

21 L. Lavelle, Psychologie et spiritualité, op. cit., p. 219.

22 II, L. 226, p. 284.

23 I, L. 55, p. 171. 
glorieuse, l'ennui est une figure du temps [...] le temps s'épaissit, s'allonge, $\mathrm{s}^{\prime}$ étire comme un bâillement $[\ldots] .^{24}$

Si l'ennui chez Mme de Graffigny vient en partie de la sensation pénible du temps, il naît aussi d'une sensation d'étouffement relative aux lieux. L'ennui possède son « territoire» ${ }^{25}$ : $c^{\prime}$ est, pour paraphraser Alain Corbin, «le territoire du vide». ${ }^{26}$ Régnant tous les jours, il possède même son propre château : " Hier Mde de Lenoncour arrive pour le dîner au château ${ }^{27}$ de l'ennui $»^{28}$ En effet, alors que l'ennui chez Mme du Deffand et Mlle de Lespinasse est essentiellement un mal de l'âme, il prend chez Mme de Graffigny la forme d'un mal des lieux. L'ennui des lieux n'est certainement pas un concept nouveau au XVIII siècle. Plusieurs théoriciens des jardins, tels que Watelet et Lezay-Marnésia, parlent de l'ennui du jardin à la française, qui ennuie à cause de sa symétrie, de son uniformité et de sa carcéralité. ${ }^{29}$ Madame de Graffigny a contre son espace les mêmes griefs. Une chambre qui n'est pas luxueuse lui devient ennuyeuse, et même les murs ${ }^{30}$ de sa chambre, qui symbolisent sans doute la claustration, sont source d'ennui: « je t'avoue que j'avais besoin de cette dissipation. Les murs de ma chambre commençaient à m'ennuyer " ${ }^{31}$ Sans doute, l'espace graffignien est-il propice à l'ennui. De toute évidence, le lieu joue un rôle primordial dans le développe-

24 Anne Wallemacq, L'Ennui et l'agitation : figures du temps, Bruxelles, De Boeck Université, 1991, quatrième de couverture.

25 «Le territoire de l'ennui » : c'est le titre du chapitre I de L'Ennui ordinaire : essai de phénoménologie sociale de Véronique Nahoum-Grappe, op. cit.

26 Voir Alain Corbin, Le Territoire du vide : l'Occident et le désir du rivage, 1750-1840, Paris, Aubier, 1988.

27 Au « château de l'ennui » dont parle Mme de Graffigny correspond le « vaste château dont l'ameublement seul [...] donnerait des vapeurs » dont parle Mme de Genlis (Adèle et Théodore ou, Lettres sur l'éducation, Paris, M. Lambert, 1782, p. 141).

28 I, L. 192, p. 60.

29 Jean Deprun, La Philosophie de l'inquiétude en France au XVIII siècle, Paris, Librairie philosophique Vrin, 1979, p. 48-49.

30 Dans ce contexte, Émile Tardieu mentionne l'importance des murs de la maison comme élément de l'ennui dans la famille: "Les murs de la maison familiale sont étouffants encore et font prison pour les actifs, remuants, préoccupés d'un but, et pour tous les agités, épris d'en avant et d'aventures, de modes nouvelles et de changements [...] » (L'Ennui : Étude psychologique, op. cit., p. 97).

31 II, L. 155, p. 49. 
ment et l'aggravation de sa maladie. Elle le sait d'ailleurs bien, comme elle l'explique elle-même à Devaux, «[s]on cher Panpan »: ${ }^{32}$

Tu n'as cessé de me gronder sur mon ennui et ma tristesse que quand tu as vu que, loin de m'en tirer, tu y joignais l'humeur : et toi tu passes ta vie à t'ennuyer et à être triste. Je veux une bonne fois faire la comparaison de notre vie afin que tu sentes une fois que je ne suis pas plus déraisonnable que toi, et beaucoup moins. Je suis à Paris, c'est une belle idée. Mais comment suis-je ? D'abord dans une maison sans comparaison plus ennuyeuse que celle de ton père et où j'étais obligée à bien plus d'égards. Tu gis à ta chambre presque autant que tu veux et très à ton aise ; car, pour nous, il faut compter les aises pour quelque chose. ${ }^{33}$

"Une maison ennuyeuse » : telle est la première cause de l'ennui et de la tristesse chez Mme de Graffigny. La noirceur ${ }^{34}$ de l'esprit passe donc avant tout par une noirceur spatiale qui opprime et impatiente. Chez Julie de Lespinasse, c'est la passion qui nourrit l'ennui; chez Mme de Graffigny, l'ennui est avant tout alimenté par l'espace qui l'entoure. Dans ce cas, il s'installe dans la scène avant d'infecter l'âme de l'héroïne. Son caractère virulent dépend strictement des conditions scéniques. On peut par conséquent affirmer que l'ennui graffignien joue le rôle d'un véritable décor ; il s'agit de cette «boîte noire ${ }^{35}$ » dont parle Didier Nordon, qui engloutit sa victime et anéantit la joie en déclenchant des manifestations morbides. Véronique Nahoum-Grappe décrit les méfaits de ce décor de l'ennui :

Le décor approprié de l'ennui, cet espace obscurci, limbique où gisent des objets défigurés autour d'un ennuyé qui devient lui-même chose, peut préexister à l'ennui éprouvé. Il peut, sur le mur d'une prison, sur une berge sinistre, dans un terrain vague pelé, entourer le promeneur de sa puissante métaphore matérielle. Ce dernier risque alors de basculer dans l'ennui sans cause, suintant tout autour, issu de la spirale des choses pesant de tout leur vide. ${ }^{36}$

32 I, L. 31, p. 47.

33 II, L. 154, p. 45.

34 Il est rare que Mme de Graffigny apparaisse gaie. Elle se montre, au contraire, toujours noire : «Ah, mon Dieu, que je suis noire! » (IV, L. 529, p. 169); « [le D.] ne put jamais m'ôter le noir qui s'était emparé de moi » (III, L. 390, p. 194).

35 "L'ennui, c'est la boîte noire ", affirme Didier Nordon dans "L'ennui, théoricien irréfutable ", Esprit, n²37, 88-98, p. 88.

36 Véronique Nahoum-Grappe, L'Ennui ordinaire: Essai de phénoménologie sociale, op. cit., p. 105. 
En tant que décor, l'ennui s'investit d'une signification matérielle. Présent dans tous les éléments du décor, il uniformise tout autour de lui : le cadre spatial où il se trouve, les choses, les paroles des personnages, le vécu quotidien. Il ruisselle de partout. Pour Mme de Graffigny, s'isoler dans sa chambre est source d'ennui; être en compagnie lui inspire aussi de l'ennui. Parler ou jouer aux cartes avec quelqu'un lui cause également de l'ennui. Peu importe le type de distraction : le mal est toujours là, envahissant son territoire et stérilisant ${ }^{37}$ son espace public ou privé. Même dans le " palais enchanté » du couple formé par Voltaire et Mme du Châtelet, elle est confrontée à l'ennui : «Tu ne croirais pas, mon cher Panpan, que les vapeurs puissent venir me trouver dans ce palais enchanté. J'en ai été accablée $" .{ }^{38}$ Maison ou palais : l'espace graffignien s'avère toujours favorable à l'incubation de l'ennui, qui s'empare du décor et prive l'endroit de son confort et de son intimité. Dans ce contexte, il est certain que l'ennui dont souffre Mme de Graffigny est intimement lié à l'extériorité, à l'entourage, à l'espace habité. Et c'est ainsi qu'en décrivant son mal, elle n'hésite pas à se servir d'indications topologiques variées : château de l'ennui, maison ennuyeuse, murs de l'ennui, palais des vapeurs, etc. Elle a même recours aux accessoires décoratifs, tels qu'un lit, ${ }^{39}$ une fenêtre ou un cabinet, pour le fuir temporairement : "Je suis honteuse de tout ce que je vais te mander, écrit-elle à Devaux ; je passai hier le reste de ma journée dans des vapeurs de tristesse si fortes que j'étais obligée de me mettre à la fenêtre à tout moment pour m'empêcher de fondre en larmes $»{ }^{40}$ Ailleurs, elle ajoute: «Quand cela m'ennuie, comme il arrive souvent, [la duchesse de Richelieu] me laisse la liberté de passer dans mon cabinet où je reprends haleine $\gg .{ }^{41}$ L'espace $a$ ainsi une double fonction : $s^{\prime}$ il est surtout cause d'ennui, il peut aussi jouer le rôle de tranquillisant temporaire.

37 Henri Lafon évoque la stérilité des paysages de la mélancolie et de la tristesse. Voir Les Décors et les choses dans le roman français du dix-huitième siècle de Prévost à Sade, Oxford, Voltaire Foundation, 1997, p. 389.

I, L. 63, p. 224.

"[...] Je suis si fatiguée et si ennuyée que je ne puis attendre d'être dans mon lit » (III, L. 475, p. 412).

40 II, L. 156, p. 54.

41 II, L. 147, p. 8. 
L'espace est donc toujours présent dans la narration de l'ennui chez Mme de Graffigny. Plus précisément, " ennui » et " espace » (comme " ennui » et " temps ») coexistent et s'entrelacent. Une telle alternance est hautement significative dans la progression de la maladie : le sentiment $d^{\prime}$ incarcération et $d^{\prime}$ instabilitét ${ }^{42}$ spatiales évolue et se transforme en ennui de l'existence : "Je ne vis point, je végète, et je végète tristement. C'est une terrible chose que de passer de maisons en maisons étrangères $\gg .{ }^{43}$

Si l'ennui résulte d'une forte sensation de pesanteur temporelle, ainsi que d'un "négativisme spatial», ${ }^{44}$ il vient aussi des personnes. Chez Mme de Graffigny les personnages de la scène quotidienne apparaissent comme serviteurs de l'ennui. Mondaine, activement engagée dans la haute société parisienne, Mme de Graffigny contracte pourtant l'ennui lorsqu'elle se trouve en compagnie des autres, particulièrement ceux qu'elle n'aime pas - car " assurément, on ne s'ennuie pas avec ce que l'on adore $»{ }^{45}$ Dans ce contexte, les visites de La Mandre l'ennuient; la mauvaise compagnie de La Taupe lui fait verser des larmes d'ennui; les dîners chez Mme de Schindek sont " de pur ennui "; ${ }^{46}$ les tête-à-tête avec Orgon lui causent une tristesse accablante. Bref, l'apparition de chaque personnage entraîne automatiquement l'apparition de l'ennui. Le lexique de l'ennui foisonne dans la Correspondance, à la mention de chaque nom propre :

42 Dans son article "Travel and the Exorcism of Boredom ", George Woodcock étudie l'ennui qui vient des déplacements et des voyages: "the traveller is inevitably lonely $[\ldots]$. The traveller has changed [...] into the diffident, self-deprecating figure of the contemporary traveller, worming his way out of misfortune and tricky situations. The travel writer is no longer the vanguard of conquest; he is increasingly the wry messenger of nostalgia. For there has been a profound change in his terrain and in his way of approaching it " (Queen's Quarterly, vol. 101, $\mathrm{n}^{\circ} 2$, summer 1994, 269-276, p. 274-275).

43 I, L. 93, p. 326.

44 Abdelkhaleq Jayed, "L'espace de l'ennui ou l'ennui de l'espace? », Bulletin des Amis d'André Gide, vol. 26, n¹18, Avril 1998, 207-227, p. 211.

45 III, L. 408, p. 244.

46 III, L. 473 , p. 409. 
Tableau 1 : Panorama des personnages qui inspirent de l'ennui

\begin{tabular}{|c|c|c|}
\hline Personnages & Citations & Références \\
\hline La Mandre & $\begin{array}{l}\text { Devine qui est encore tombée des nuës ici pour } \\
\text { m'ennuyer. La Mandre. }\end{array}$ & I, L. 33, p. 58 \\
\hline La Taupe & $\begin{array}{l}{[\ldots] \text { l'ennui m'avait surmontée parce que La Taupe }} \\
\text { n'avait jamais été si mauvaise compagnie }[\ldots] \text {. }\end{array}$ & I, L. 43, p. 105 \\
\hline Doroté & $\begin{array}{l}{[\ldots] \text { l'ennui }[\ldots] \text { que me donna la longue visite du }} \\
\text { mai de Doroté }[\ldots] \text {. }\end{array}$ & I, L. 109, p. 403 \\
\hline Orgon & Cet Orgon m'ennuie les soirs à crever [...]. & II, L. 254, p. 351 \\
\hline Lucile & $\begin{array}{l}\text { [...] je n'ai eu que de l'ennui. Je fus lundi chez } \\
\text { Lucile dans l'intention de m'ennuyer et je remplis } \\
\text { bien ma vocation. }\end{array}$ & II, L. 258, p. 366 \\
\hline Ragonde & $\begin{array}{l}\text { [...] qui me mena chez Ragonde dont je ne suis } \\
\text { revenue qu'à minuit, bien ennuyée et pleine de } \\
\text { vapeurs. }\end{array}$ & IV, L. 521, p. 130 \\
\hline La Cousine & $\begin{array}{l}\text { J'ai été dîné chez la Cousine aujourd'hui, où je me } \\
\text { suis fort ennuyée. }\end{array}$ & IV, L. 557, p. 263 \\
\hline Doris & $\begin{array}{l}\text { Elle est en vérité charmante pour moi, cette femme, } \\
\text { et elle m'ennuie. }\end{array}$ & III, L. 400, p. 227 \\
\hline Julie & $\begin{array}{l}\text { J'ai dîné hier chez Julie. [...] J'en suis revenue } \\
\text { avec un mal de tête et une fatigue horrible de pur } \\
\text { ennui. }\end{array}$ & III, L. 473, p. 409 \\
\hline Romagnesi & $\begin{array}{l}\text { Je détestais Romagnesi. C'est de ces gens que tu } \\
\text { sais que je souhaite mort, tant ils m'ennuient [...]. }\end{array}$ & III, L. 486, p. 450 \\
\hline $\begin{array}{l}\text { Mme } \\
\text { Herbert }\end{array}$ & $\begin{array}{l}\text { [...] J'envoyai demander la carrosse de Mme Her- } \\
\text { bert. Je dînai chez elle, dieux, quel ennui ! }\end{array}$ & II, L. 264, p. 378 \\
\hline $\begin{array}{l}\text { Une abbesse } \\
\text { sœur }\end{array}$ & $\begin{array}{l}\text { Je fus dîner indispensablement avec une autre } \\
\text { abbesse sœur. [...] Je m'ennuyai, Dieu sait. }\end{array}$ & II, L. 298, p. 436 \\
\hline Duvigeon & $\begin{array}{l}\text { Ma Sœur m'a ennuyée, et Duvigeon encore plus } \\
{[\ldots] .}\end{array}$ & I, L. 123, p. 461 \\
\hline $\begin{array}{l}\text { La petite } \\
\text { sœur }\end{array}$ & $\begin{array}{l}\text { [...] je sortais de m'ennuyer comme un chien chez } \\
\text { Mon Amie à cause de cette petite sœur qui est ma } \\
\text { chenille. }\end{array}$ & II, L. 177, p. 127 \\
\hline
\end{tabular}


Chez cette « créature la mieux ennuyée de tout le royaume $"{ }^{47} l^{\prime}$ ennui agit comme un véritable décor animé, suivant les va-et-vient des personnages. Peu importe l'âge, la personnalité et la grandeur d'esprit, toute personne est à l'origine du mal qu'elle diffuse autour d'elle. À cet égard, qu'elle soit vieille ou jeune, ${ }^{48}$ charmante ou désagréable, la femme cause toujours de l'ennui : "Je ne te dirai pas grand-chose ce soir, mon ami. J'ai eu du monde tout le jour et des femmes, par conséquent, de l'ennui. Il m'a saisi au point que je me suis trouvée mal ". ${ }^{49}$ $\mathrm{Du}$ reste, la présence masculine engendre aussi l'ennui. "Ce chien d'ennui $»^{50}$ saisit Mme de Graffigny en compagnie du sage Orgon ${ }^{51}$ ou lors d'une rencontre avec «le méchant étranger» qui la fait "[périr] d'ennui, de honte et de fatigue $»{ }^{52}$ Hommes, femmes et enfants, ces personnages innombrables du quotidien, "qui [1]'ennuient plus qu'ils ne [l]'amusent ${ }^{53}$ ne parviennent qu'à rendre son mal de plus en plus insupportable. Qu'est-ce donc que ce monde? C'est bien sûr « le monde de l'ennui ", répond Jean Bloch-Michel; c'est

ce monde sans communication possible, ni avec les êtres ni avec les choses. [...] Ce monde de la "parlerie » est précisément caractérisé par l'absence de communication entre ceux qui parlent. Car le langage n'y établit entre les êtres aucun rapport profond. D'une certaine manière, le langage y devient un moyen de $\mathrm{s}^{\prime}$ isoler plutôt qu'un moyen de se rencontrer, plutôt un rempart qu'un pont. ${ }^{54}$

47 I, L. 109, p. 404.

48 Les jeunes filles l'ennuient aussi : « aujourd'hui, je n'ai encore vu que des nones et ces petites filles qui m'ennuient » (III, L. 390, p. 194).

49 III, L. 389, p. 192.

50 III, L. 390, p. 194.

51 «Ce pauvre Orgon, tout plein d'esprit, le jugement fin, pénétrant, délicat, rempli de sagacité, le cœur excessivement humain, quoique souvent excessivement dur, m'ennuyait à périr au bout d'une demi-heure, quelquefois avant » (IV, L. 496, p. 27).

52 IV, L. 495, p. 19.

53 IV, L. 553, p. 251.

54 Jean Bloch-Michel, Le Présent de l'indicatif : essai sur le nouveau roman, Paris, Gallimard, 1963 (édition revue et augmentée : Gallimard, 1973), p. 62-63. En fait, Mme de Graffigny est consciente de ce manque de communication qui caractérise ses cercles de conversations pures. Ainsi dit-elle à son ami Devaux : "Le commerce des amis que j'ai ici me plairait peut-être mieux en lettres qu'en conversation, mon cœur n'y tint pas enfin, ou du moins ce sont de ces liens formés par l'estime, la 
Chez Madame de Graffigny, l'ennui est donc lié aux différents aspects de la vie quotidienne : temps, espace et personnages sont au service du mal graffignien. Dans sa correspondance, elle ne cesse de révéler les causes de son ennui, qu'on pourrait considérer comme des accessoires de l'ennui, étant donné leur aspect matériel. Ces accessoires peuvent par exemple prendre la forme d'une longue attente, comme celle d'un " maudit carrosse ${ }^{55}$ ou d'un courrier en retard ${ }^{56}$ Parfois encore, les accessoires de l'ennui correspondent aux diverses sortes de divertissement. Chez Madame de Graffigny, le remède à l'ennui est inutile : bien souvent ce remède lui-même est cause d'ennui.

Rien ne peut vaincre l'ennui. Chez Mme de Graffigny, le mal apparaît incurable; il vient du bien et du mal, des douleurs comme du divertissement. Aucune consolation, aucun soulagement: l'ennui s'intègre dans toute activité et toute distraction. Il "semble une puissance maléfique, une présence, [...] une figure détestable.» ${ }^{57} \mathrm{Chez} \mathrm{Mme}$ de Graffigny, être seule ou en compagnie, vivre en ville ou passer des jours à la campagne, aller à la messe ou jouer au trictrac déclenchent le même mal : l'ennui. Qu'elle décrive ses joies ou ses tristesses, elle murmure toujours la même plainte : «je m'ennuie à mourir ». ${ }^{58}$

Omniprésent, l'ennui est un redoutable compagnon. Bien qu'il s'agisse d'un mal et d'un état d'esprit, cet ennui peut en effet être personnifié. ${ }^{59}$ Vladimir Jankélévitch en est conscient lorsqu'il soutient que «l'ennui apparaît comme une créature entre toutes protéiforme : il a

convenance, la nécessité de voir quelqu'un, mais il n'y en a aucun formé par le goût, et aucun qui ne m'ennuie » (IV, L. 496, 27).

55 « Je croyais que mon amie m'envoierait chercher ce matin. Point du tout. Ma journée a été destinée de toute éternité au plus profond ennui. Tout le jour s'est passé à attendre le maudit carrosse qui n'est point venu. Tu sais comme on est quand on attend » (I, L. 144, p. 550).

56 «Bonjour, mon Penpichon. Je ne me porte pas si bien aujourd'hui que je me vantais hier. Je ne sais si ce sont tes lettres non reçues qui m'ont donné des vapeurs, mais enfin j'en ai eu hier soir " (I, L. 62, p. 210); «Bonsoir, la poste va arriver. Je l'attends en tremblant; il y a plus d'une heure que j'en ai des vapeurs » (I, L. 63, p. 217).

57 Élisabeth Ravoux-Rallo, "Variations sur l'intranquillité ", Revue de Littératures Française et Comparée, vol. 9, 1997, p. 271.

58 II, L. 230, p. 298.

59 Selon Michel Gilot et Jean Sgard, Loaisel de Tréogate personnifie l'ennui dans Dolbreuse (1785, I, p. 130) : " ...et l'ennui resserrant et fermant mon cœur, l'entoura d'une enveloppe qui devait bientôt se briser, en le déchirant de mille blessures " (cité par M. Gilot et J. Sgard, "La vie intérieure et les mots », dans P. Viallaneix 
Tableau 2 : «Divertissements » qui contribuent à l'ennui graffignien

\begin{tabular}{|c|c|c|}
\hline Divertissement & Citations & Référence \\
\hline L'opéra & 0 qu'il m’a ennuyée cet opéra. & I, L. 102, p. 369 \\
\hline La compagnie & Oh, dame, la compagnie $[\ldots]$ m'ennuyait $[\ldots]$. & III, L. 397, p. 217 \\
\hline La campagne & $\begin{array}{l}\text { J'arrive de la campagne où je n'ai trouvé de res- } \\
\text { sources contre l'ennui que la promenade. Ainsi je } \\
\text { suis excédée de l'un et de l'autre. }\end{array}$ & III, L. 422 , p. 272 \\
\hline \multirow[t]{2}{*}{ Le jeu } & $\begin{array}{l}\text { La soirée se passa à jouer au trictrac qui m'ennuie } \\
\text { à présent à cause de la cause; }\end{array}$ & I, L. 129, p. 486 \\
\hline & $\begin{array}{l}\text { Je passais la journée à jouer au piquet, c'est-à- } \\
\text { dire que je m'ennuyai }[\ldots] \text {. }\end{array}$ & III, L. 460, p. 360 \\
\hline La solitude & $\begin{array}{l}\text { [...] Aujourd'hui, j'étais destinée de toute éternité } \\
\text { à être seule. [...] moi qui sens aussi bien que toi } \\
\text { le prix d'une journée donnée à la jouissance de } \\
\text { soi-même, je me suis avisée de le trouver mau- } \\
\text { vais, soit vapeurs que j'avais ou que ces vapeurs } \\
\text { vinssent de là. }\end{array}$ & IV, L. 572, p. 322 \\
\hline L'Église & $\begin{array}{l}{[\ldots] \text { j'ai été depuis neuf heures à l'église }[\ldots] . . .} \\
\text { avait une profession chez nous qui m'a excédée } \\
\text { d'ennui. }\end{array}$ & III, L. 386, p. 185 \\
\hline
\end{tabular}

mille visages contradictoires, parle mille langages, entre comme ingrédient dans une foule de complexes.» ${ }^{60} \mathrm{Il}$ se transforme alors en méchant héros, toujours au rendez-vous pour embêter sa proie. Ce redoutable personnage, Mme de Graffigny y est confrontée en tout temps, tout lieu et toute circonstance; il la poursuit sur toutes les scènes de ce théâtre qu'est la vie mondaine. D'ailleurs, Mme de Graffigny reconnaît elle-

(actes établis et présentés par), Le Préromantisme : Hypothèque ou Hypothèse?, Paris, Klincksieck, 1975, 509-528, p. 515).

60 V. Jankélévitch, L'Alternative, op. cit., p. 127. 
même l'aspect actif, dynamique et mobile de son adversaire: « À ta lettre d'aujourd'hui, tu t'ennuies donc aussi, mon ami. C'est un méchant vent qui l'apporte cet ennui : il vient par bouffée $»{ }^{61}$ Nuisible et contrariant, l'ennui freine l'action. Quand il s'en prend à Mme de Graffigny, il dérange toujours ses habiletés et ses activités, l'empêche même d'écrire des lettres à " [s]on Penpichon »: $:^{62}$ "Il n'est pas possible de pousser l'ennui plus loin que je l'ai hier poussé. C'est ce qui m'a empêché de t'écrire ${ }^{63}{ }^{63}$ En outre, l'ennui a un effet néfaste sur la santé : il cause non seulement à Mme de Graffigny des langueurs d'estomac et des problèmes de digestion, mais aussi de la fatigue et des maux de tête. ${ }^{64}$ Les vapeurs sont encore une réaction négative à l'ennui. Grande ennuyée, Mme de Graffigny est aussi une grande vaporeuse. Chez elle, l'état d'esprit dépend toujours de l'état physique, et vice versa ; l'ennui s'accompagne de douleurs physiques et de convulsions nerveuses, semblables à la folie. Dans sa correspondance, elle fait souvent le lien entre «ennui " et "vapeurs " ${ }^{65}:$ "Je ne sais ce que je vais t'écrire, mon ami. Je bouffe d'ennui et de vapeurs "; " Eh mon Dieu, voilà la manivelle des vapeurs et de l'ennui. Je l'avais oublié » ${ }^{66}$

Comme Mlle de Lespinasse et Mme du Deffand, Mme de Graffigny est victime d'une maladie rebelle qui bouleverse l'être et le paraître, qui cause le chaos aussi bien dans la tête que dans la vie entière. L'ennui vient à la rencontre de la souffrante. Il transforme les désirs en douleurs, l'activité en passivité, la vie en véritable cauchemar; pouvoir perturbateur, il sème le désordre et entraîne la paralysie. Il s'oppose au développement de l'action en condamnant sa victime à l'inertie et à l'assoupissement. Comme le dit le vicomte de * au duc dans Les Malheurs de l'inconstance de Dorat : "quand on s'ennuie, on n'a la force de rien $»{ }^{67}$ Aux moments d'ennui, Mme de Graffigny ne peut ni lire, ni écrire, ni penser. Elle a recours au sommeil comme moyen d'évasion

III, L. 390, p. 195.

62 I, L. 109, p. 403.

63 I, L. 34, p. 61. I, L. 38, p. 80; I, L. 31, p. 47; III, L. 473, p. 409. «Il me vint Thiriot d'abord après dîner, et Mr. De Tavannes qui me mena chez Ragonde dont je ne suis revenue qu'à minuit, bien ennuyée et pleine de vapeurs. Je n'ai guère dormi » (IV, L. 521, p. 130); " La tête me tourne d'ennui et de vapeurs, mon cher ami » (III, L. 402, p. 232).

66 IV, L. 572, p. 325.

67 Dorat, Les Malheurs de l'inconstance, Paris, Desjonquères, 1983, p. 75. 
temporaire. Le lit apparaît, dans ce cas, comme l'objet principal du décor de l'ennui :

J'avais hier tant de vapeurs et mon hôtesse fut si ennuyeuse l'après-souper que je ne pus que me jeter dans mon lit. ${ }^{68}$

On ne peut ignorer l'originalité de l'ennui graffignien. Alors que chez Julie de Lespinasse, l'ennui se fortifie par l'imaginaire qui procure un univers propice, chez Mme de Graffigny la nouveauté du mal réside dans son rapport avec le théâtre. Pour elle, l'environnement se présente comme un théâtre. La progression de l'ennui graffignien dépend ainsi de facteurs purement théâtraux: temps, lieux, personnages, décor et accessoires. Il en résulte une représentation théâtrale de l'ennui. Occupant toujours la scène quotidienne, l'ennui chez Mme de Graffigny n'a apparemment rien de tragique; étant plus lié à l'extériorité qu'à l'intériorité, il « se constitue en spectacle [...] [et] domine tous les éléments de l'espace tristement vide où la vie s'égoutte et se perd irrémédiablement.» ${ }^{69}$ Sa pesanteur dépend du vide quotidien. Son évolution suit de près l'évolution journalière. Ses principales causes naissent des diverses incommodités de la vie. Bref, son intrigue correspond à celle de la vie. Il s'agit d'un mal qui se présente comme le dénouement du spectacle de la mondanité.

La correspondance de Mme de Graffigny dévoile clairement «les deux visages de l'ennui.» ${ }^{70} \mathrm{Du}$ théâtral au tragique, de l'extérieur à l'intérieur, du corporel au spirituel, l'ennui graffignien s'aggrave et subit progressivement une saisissante métamorphose. Perpétuel, il se transforme en "ver solitaire», ${ }^{71}$ selon la terminologie de Mme du Deffand, torturant sans pitié l'âme de la malade. En effet, si Mme de Graffigny fait au premier abord la description d'un ennui qui, loin d'être

68 I, L. 37, p. 71. «[...] J'avais donc commencé, ayant déjà un peu de vapeurs, malgré le plaisir que j'avais. Elles ont tellement augmenté qu'il a fallu que je me jette sur mon lit » (I, L. 63, p. 224); "Quelquefois j'ai bien la peine à tenir le temps de la table. Je me trouve mal, cela fait du train par le soin qu'on a de moi. On me retraine dans ma chambre, on me jette au lit. Voilà bien ma vie : elle m'est insupportable à cause de l'ennui que je cause que par le mal que j'en souffre » (I, L. 75, p. 275).

69 Abdelkhaleq Jayed, «L'espace de l'ennui ou l'ennui de l'espace? », loc. cit., p. 210.

70 C'est le titre d'un article de Charles Richet, "Les deux visages de l'ennui », Revue des deux mondes, 15 juillet 1932, 313-324.

71 Lettres de Madame Du Deffand, Paris, Mercure de France, 2002 : lettre du 7 février 1773, p. 556. 
amer, symbolise l'impatience et la mauvaise humeur ${ }^{72}$, elle parle aussi d'un ennui violent qui ressemble fort à celui de Mme du Deffand ou de Julie de Lespinasse, d'un "ennui morbide» ${ }^{73}$ qui se débarrasse de ses valeurs quotidiennes pour devenir un sentiment $d^{\prime}$ abattement ${ }^{74}$ et de tristesse accablante :

Je pensais t'écrire hier au soir pour te dire combien j'étais ennuyée, mais ma lettre n'aurait été que des exclamations. [...] Je ne sais ce que c'est que cette fièvre lente si ce n'est l'accablement de tristesse qui ne me quitte pas. ${ }^{75}$

Mme de Graffigny ne ressent désormais plus la joie de l'âme. ${ }^{76}$ Condamnée à un ennui étouffant, elle s'abandonne involontairement à la tristesse. C'est une crise qui semble sans fin. Et la malade, à un degré plus profond de son mal, reste dans un état de prostration permanent ${ }^{77}:$ " Je $\mathrm{n}^{\prime} \mathrm{y}$ vis que le fond de tristesse qui me domine ${ }^{78}{ }^{78}$ Cette tristesse qui la domine obscurcit son âme et entraîne irrémédiablement la perte de la gaieté intérieure :

Voyons ta lettre. Tu te portes bien, mon ami : j'en suis si aise, si aise que j'en serais gaie si je n'étais pas triste, car je le suis toujours. C'est une besogne toisée ; il n'y faut plus penser. C'est cette ancienne maladie dont je te parle depuis

72 Mme de Graffigny apparaît toujours de mauvaise humeur : « Je suis de bien mauvaise humeur " (II, L. 207, p. 228); " Je suis d'une humeur de chien » (III, L. 484, p. 443); " Tu es déjà de méchante humeur, mon ami. Eh mon Dieu, moi, j'en suis toujours, et je suis dans les excès " (III, L. 381, p. 170).

73 Dans son article «L'ennui morbide", L. Dupuis justifie bien cette évolution de l'ennui : "S'il est vrai [...] qu'un sentiment devienne pathologique par l'exagération de sa durée et de son intensité et par sa disproportion aux circonstances, une étude de l'ennui morbide suppose une détermination préalable de l'ennui normal » (Revue philosophique, v. 93, 1922, 417-442, p. 417).

74 " [...] Cela me jette dans l'abattement que tu sais [...] ce n'est jamais le corps qui m'occupe" (III, L. 381, p. 170).

75 II, L. 247, p. 335.

76 Ni joie, ni espoir de l'âme : « [...] mon âme ne sait plus espérer » (I, L. 93, p. 326).

77 Les citations qui témoignent de cette permanence de la tristesse abondent : «Si j'étais comme autrefois, j'aurais éprouvé aujourd'hui le passage de la plus vive tristesse à la joie la plus marquée. Hélas, mon ami, j'éprouve encore qu'il n'en est plus pour moi » (IV, L. 533, p. 184); « Mets-toi dans la tête qu'il n'y a plus de plaisir pour mon cœur. Il est fermé pour jamais à toutes idées agréables » (III, L. 369, p. 128).

78 III, L. 391, p. 199. 
si longtemps. Ma gaieté intérieure est partie sans retour. Il n'y plus que mon visage qui rit et par habitude [...]. Je croyais que c'était les embarras enchaînés les uns aux autres qui me mettaient dans cet état, mais je vois à présent que cela est incurable. $^{79}$

Si Mme de Graffigny agit sans joie, elle connaît aussi un épuisement mental. Cette tristesse de l'âme, forme aiguë de l'ennui, retentit sur l'esprit. Elle affecte jusqu'aux activités intellectuelles. Manque d'idées, manque d'anecdotes, manque d'éloquence, manque d'imagination ${ }^{80}$ : tels sont les signes évidents d'une stérilité intellectuelle, reflet de l'inertie de l'âme :

Je ne t'écrivis pas hier, mon ami, précisément faute de savoir que dire. Je m'étais ennuyée complètement toute la journée. Tu sais l'effet que l'ennui fait sur moi. Je n'avais point de faits à te conter, et il faut de l'esprit pour raisonner, même pour dire les amitiés comme tu fais ; car je trouve que ton éloquence sur ce chapitre est inépuisable. Pour moi, je n'ai ni esprit ni éloquence ni.... ni... ${ }^{81}$

Que te dirai-je encore? Rien, car je ne sais rien. Je suis dans la profonde ignorance, et la plus insipide sécheresse. ${ }^{82}$

Maladie de l'âme, ${ }^{83} l^{\prime}$ ennui apparaît aussi comme un «tourment d'esprit». ${ }^{84}$ Il est sûr que l'ennui chez Mme de Graffigny provoque la déchéance fonctionnelle de l'esprit. Elle le sait d'ailleurs, puisqu'elle ne

79 IV, L. 510, p. 86.

80 " $[\ldots]$ tu dis qu'il me faut dissiper. Avec quoi, dis donc? Avec mon imagination, plus noire que jamais » (IV, L. 519, p. 121).

81 I, L. 123, p. 461.

82 III, L. 393, p. 207

83 « Mon âme est bien plus malade que mon corps » (IV, L. 529, p. 169).

84 III, L. 398, p. 225. On retrouve cette définition de l'ennui en tant que mal de l'esprit chez Jacques Autreau (1657-1745), dans cet extrait tiré de La Fille inquiète, acte I, scène 7 (dans la Petite Bibliothèque des théâtres, Paris, 1785, p. 19) :

Lisette : Êtes-vous lasse? Vous sortez du lit; qu'avez-vous donc?

Silvia : Je ne sais.

Lisette: N'est-ce point que vous vous trouvez mal?

Silvia: Oui, j'ai mal à l'esprit.

Lisette : Qu'appelez-vous, s'il vous plaît, mal à l'esprit?

Silvia : Belle demande! De l'inquiétude, de l'ennui, de la langueur, que sais-je! 
cesse d'avouer, en toute honnêteté et lucidité : « Je suis bête, tu sais ce que je suis quand l'ennui surmonte $\gg .^{85}$

La sécheresse mentale qu'éprouve Mme de Graffigny dans les moments d'ennui n'est pas seulement visible sur le plan du psychisme intellectuel, elle affecte aussi sa vision générale de l'existence. Si Mme de Graffigny perd la joie de l'âme, elle perd aussi la joie de vivre. Exprime-t-elle alors une conception lespinassienne de la vie? On croit entendre la voix de Julie de Lespinasse, criant dans le désert de son âme : "Mon Dieu, que je suis lasse de vivre ». ${ }^{86}$ Les définitions singulièrement aiguës de la vie réapparaissent sous la plume de Mme de Graffigny. La vie se définit tantôt comme un fardeau, tantôt comme une souffrance, une suite de malheurs et de " peines réelles" ${ }^{87}$ qui commence le jour de la naissance : «Vois-tu, mon ami, demande-t-elle à Devaux, la date du plus malheureux jour de ma vie, puisque c'est celui de ma naissance ${ }^{88}$ Ailleurs, Mme de Graffigny suit l'inclination racinienne de Julie de Lespinasse lorsqu'elle assimile sa vie à une tragédie : "Je n'ai nulle espérance que celle de voir finir bientôt la tragédie de ma vie, et je doute que je n'approche de la catastrophe. Ce jour-là ne sera sûrement pas le plus douloureux $» .^{89}$ Or si la vie est une tragédie, le meilleur dénouement ne serait-il pas la mort ? À l'instar de Julie de Lespinasse, Mme de Graffigny voit en effet la mort ${ }^{90} \mathrm{~d}^{\prime} \mathrm{un}$ bon œil. Plus son dégoût de la vie augmente, plus sa peur de la mort diminue. L'ennui de la vie la pousse à accueillir la mort à bras ouverts :

II, L. 235, p. 310.

«Écoute. Personne dans le monde ne compatit plus que moi aux peines réelles " (III, L. 371, p. 132); « Mes peines sont toujours les mêmes, et ne finiront qu'avec ma vie » (III, L. 438, p. 307).

III, L. 356, p. 96.

III, L. 361, p. 111.

90 English Showalter décrit l'importance de la mort chez Mme de Graffigny : " Depression and anxiety surely made her situation worse, but, as for most of her contemporaries, sickness, pain and death were never far from her thoughts. [...] Mme de Graffigny had lived through an astonishing number of upheavals produced by the premature deaths of public figures alone » (Françoise de Graffigny, Her life and works, Oxford, Voltaire Foundation, 2004, p. 107). 
Je me trouve encore bien plus isolée ici, mes amis me paraissent bien plus absents, je ne tiens plus à rien, et la fin de ma vie est l'unique objet de mes souhaits. C'est un point de vue fixe duquel je ne puis me détourner. ${ }^{91}$

À cette confidence en fait écho une autre où, dans les moments les plus accrus de l'ennui, elle "n'envisage que la mort d'agréable $» .{ }^{92} C^{\prime}$ est le dégoût de la vie par excellence.

Ce dégoût de la vie ne marque cependant pas le bout de l'ennui graffignien; l'écoeurement est généralisé, il s'empare de tout. Dans " ces sécheresses de cœur et d'esprit qui ressemblent si bien à l'anéantissement ${ }^{93}$ Mme de Graffigny n'a le goût de rien, du goût pour rien. Tout lui inspire au contraire le sentiment d'un dégoût écrasant, qui inclut le dégoût de la vie et celui de soi : "Je sens toute ma misère, elle est insupportable. Je ne suis pas dans un état violent, mais si battu, si dégoûté de moi et de la vie que tout m'est insipide». ${ }^{94}$ Lorsque le dégoût de la vie s'aggrave, il devient dégoût des autres : "Je me hais moi-même. Comment aurai-je de l'indulgence pour les autres ». ${ }^{95} \mathrm{Ce}$ dégoût des autres signifie, bien sûr, un dégoût de l'humanité tout entière: ${ }^{96}$

Au reste, je vous prie de me laisser en repos. Je suis si détachée de tout ce qu'il $\mathrm{y}$ a de figure humaine dans le monde, leur méchanceté et leur injustice me sont si connues, que je n'en veux plus entendre parler. ${ }^{97}$

Dégoût de la vie, dégoût de soi, dégoût des autres, dégoût del'humanité : l'ennui graffignien ne signifie-t-il pas, tout simplement, le dégoût de tout ? C'est ce qu'elle confirme en une formule définitive, désespérante et désespérée : " J'ai bien éprouvé que mon cœur ne peut plus être con-

91 I, L. 93, p. 326.

92 II, L. 162, p. 78.

93 II, L. 222, p. 269.

94 I, L. 92, p. 324.

95 II, L. 172, p. 111.

96 Ailleurs, Mme de Graffigny exprime ce dégoût de l'humanité en évoquant son idée de la noirceur humaine: "On se fait à tout. J'en ai tant éprouvé, je suis si excédée de la noirceur humaine que je souffre la moitié moins que du passé. Je ne dis pas pour cela que j'y sois insensible : il s'en faut bien... » (III, L. 427, p. 281).

97 III, L. 425, p. 278. 
tent ni à son aise. Il devait l'être, et reste dans une stupidité qui me dégoûte de tout, sans exception ${ }^{98}{ }^{8}$

RAOUDHA KALLEL

Université Acadia

98 III, L. 400, p. 227; nous soulignons. Ce sentiment de dégoût de tout persiste sous sa plume. Deux autres exemples le prouvent : «[...] Je suis dans mes grands dégoûts du monde" (III, L. 407, p. 241); " Tu sais que de temps en temps le sentiment de malheur devient plus vif. Il le fut tant avant hier soir que je passai presque toute la nuit à pleurer. Hier j'étais dans mon accablement si grand et si pleurante, si dégoûtée de tout, que je fis dire que je ne voyais personne. Je restai livrée au chagrin; il fit de moi ce qu'il voulut » (II, L. 248, p. 337). 